\title{
The whirlwinds of Elysium: A catalog and meteorological characteristics of "dust devil" vortices observed by InSight on Mars
}

\author{
Ralph D. Lorenz ${ }^{\mathrm{a}, *}$, Aymeric Spiga ${ }^{\mathrm{b}, \mathrm{f}}$, Philippe Lognonnéc ${ }^{\mathrm{c}}$, Matthieu Plasman ${ }^{\mathrm{c}}$, Claire E. Newman $^{\mathrm{d}}$, \\ Constantinos Charalambous ${ }^{\mathrm{e}}$ \\ a Johns Hopkins Applied Physics Laboratory, Laurel, MD 20723, USA \\ ${ }^{\mathrm{b}}$ Laboratoire de Météorologie Dynamique (LMD/IPSL), Sorbonne Université, Centre National de la Recherche Scientifique, École Polytechnique, École Normale Supérieure, Paris, France \\ ${ }^{\mathrm{c}}$ Institut de Physique du Globe de Paris, Université de Paris, CNRS, Paris, France \\ d Aeolis Research, 333 N Dobson Road, Unit 5, Chandler, AZ 85224-4412, USA \\ e Imperial College London, Department of Electrical and Electronic Engineering, Imperial College London, South Kensington Campus, London SW7 2AZ, United Kingdom \\ ${ }^{\mathrm{f}}$ Institut Universitaire de France, Paris, France
}

\section{A R T I C LE IN F O}

\section{Keywords}

Mars atmosphere

Convective vortices

Dust devils

Statistics

\begin{abstract}
A B S T R A C T
A catalog of convective vortex encounters recorded by InSight on the Martian surface is presented, through Sol 390 of the mission. The catalog summarizes key meteorological parameters such as wind speed and direction before the event, peak wind, the duration and magnitude of the pressure excursion, temperatures and solar array data where present. Additional seismic parameters are also provided on seismometer-detected ground acceleration. The catalog is intended as a resource for vortex population studies, and as an index for examining these meteorological events in detail and to assess possible geophysical (seismic or magnetic) signatures. Whereas it is difficult to evaluate 'anecdotal' results in small surveys, the large number of events (853 with a pressure drop exceeding $0.8 \mathrm{~Pa}$ ) in this work permits robust statistical evaluation. For example, it is found that three times as many pressure profiles have slower onsets than decays than vice versa, indicating an asymmetry in the surface pressure field due to the tilted advection of the vortex by ambient wind. A vortex area fraction of $0.07 \%$ during the most active six hours of the day is deduced.
\end{abstract}

\section{Introduction}

Dust devils, and their invisible dustless vortex counterparts, are one of the most prominent, and fascinating, aspects of Martian meteorology. They have been documented by every landed mission (even those - the Mars Exploration Rovers - without dedicated meteorological instrumentation). Dust devils play important roles in injecting dust into the atmosphere (e.g. Cantor et al., 2006; Kahre et al., 2006) and causing surface albedo changes via the formation of dust devil tracks (e.g. Reiss and Lorenz, 2016). They also impact the operation of solar-powered vehicles on Mars, via the removal of airfall dust from solar panels, and are believed to be responsible for the 'cleaning' of surfaces and optics on several surface missions (e.g. Holstein-Rathlou et al., 2010; Kinch et al., 2015; Vicente-Retortillo et al., 2018; Reiss and Lorenz, 2016). A further virtue of vortices for InSight, given the apparent dearth of geological seismic events, is their role as a (negative) surface load which probes the elastic properties of the near-surface (Murdoch et al., 2017; Banerdt et al., 2020; Lognonné et al., 2020; Kenda et al., 2020).
The InSight lander has been operating on the surface of Mars since November 2018 and, as a fixed geophysical monitoring platform with meteorological instrumentation, is yielding a record of Martian weather that is transformative in its detail and uniformity (Banfield et al., 2020). Very soon after its instrumentation was checked out, transient pressure drops due to convective vortices were detected. Spiga et al., 2020 presented the detection of almost ten thousand pressure drop events through Sol 390, noting the seasonal evolution of the population in response to changing wind and insolation patterns, as well as due to the passage of a dust storm.

The present paper presents a catalog of the largest events in that same period, extracting key meteorological parameters beyond the simple pressure drop magnitude. The catalog comprises 'browse' plots of key meteorological variables over the 2 min period around the maximum pressure drop, and an ASCII table of key extracted quantities. It is not intended that strong conclusions be drawn from any single catalog entry - the original data should be reviewed over a wider period for full context, for example - but broad statistical correlations from the catalog should be valid.

\footnotetext{
* Corresponding author.

E-mail address: RALPH.LORENZ@JHUAPL.EDU (R.D. Lorenz)
} 
We show in this paper a few example plots of events to illustrate the data quality and features of the vortices. The present study also reviews some statistical aspects of the vortex population and some relationships between the vortex properties and meteorological parameters. This paper is not intended as an exhaustive investigation of all possible correlations - indeed the provision of the catalog is precisely intended to facilitate investigations that the authors have not anticipated. It is hoped that the catalog will help identify occasions where close scrutiny of other datasets may be of interest.

\section{Data acquisition and catalog generation}

The present catalog of vortex properties (access and structure of the catalog are discussed in the appendix) retrieves the local time series of all meteorological data in the $2 \mathrm{~min}$ around these events and extracts key parameters. Examples of the browse plots are shown in Figs. 1-3.

The pressure time series, being the most heavily-sampled data and most uniform in quality over the course of the mission, is used to define the vortex event, as in many previous studies. Pressure signals also tend to be the most robust vortex indicators. The detection method of InSight vortex encounters by induced pressure drops is described in Spiga et al., 2020 and Banfield et al., 2020. Although the resolution of the Auxiliary Payload Sensor Suite (APSS) pressure sensor (Banfield et al. $2019)$ is exquisite $(\sim 0.01 \mathrm{~Pa})$ the background fluctuations in pressure are typically of the order of $0.1 \mathrm{~Pa}$ and a practical detection threshold scales to some multiple of this value. Generally, the peak pressure drops reported in the catalog appear to be accurate to $5 \%$ or better, but in $5 \%-10 \%$ of cases, irregularities in the background pressure may cause the peak to be mis-estimated by up to $20 \%$ or so. In other words, the accuracy of estimating the pressure drop is not limited by the instrument precision, but rather on where or how the vortex is defined to begin and end. In this respect, however, the present survey is no different from other pressure surveys on Earth and Mars.

As noted in Spiga et al., 2020 many thousands of pressure excursions with amplitudes of 0.3 or $0.5 \mathrm{~Pa}$ (thresholds used in studies by Pathfinder (Murphy and Nelli, 2002), Phoenix (Ellehoj et al., 2010) and Curiosity (e.g. Ordóñez-Etxeberria et al., 2020) have been detected at InSight. However, the signatures in other meteorological data of such small events are typically comparable with the background fluctuations associated with convective turbulence. Thus to focus attention where vortex signatures are relatively unambiguous, the present catalog is restricted to events with a pressure drop of $0.8 \mathrm{~Pa}$ or more.

Wind data on InSight are recorded by a pair of thermal anemometers ('TWINS') pointing in the Plus and Minus Y directions in the local lander level (LL) (roughly East and West respectively). The sensors are located at the edge of the lander deck and stand at approximately $1.2 \mathrm{~m}$ from the ground, with a $10 \mathrm{~cm}$ difference between the two due to the tilt of the landed spacecraft in Homestead hollow (Banfield et al. 2019). These sensors have a sample rate of $1 \mathrm{~Hz}$ and post-processing combining the data from both sensors (if both are powered on) determines the best overall estimate of wind direction and wind speed. However, the rapidly-changing speed and direction in a vortex often defeats this estimation process and itfails to generate a valid estimate. This results in some data gaps, often frustratingly at the most interesting moment in the record. This is particularly important for the wind peaks which are most critical for inferring the friction speeds required for inducing an aeolian change on the Martian surface (Charalambous et al., 2020, Baker et al., 2020). We report, in the catalog plots, the separate intermediate products (the so-called Plus and Minus wind speeds), and (following Charalambous et al., 2020) consider the most likely 'true' value to be the higher of the two if both are available: this is the value reported in the catalog table. The retrieved wind direction tends to be retrieved more reliably than the speed. Although a full diagnosis of the wind measurement precision (which is dependent on the wind speed itself, the direction, and the turbulent variation of these quantities and temperature) is beyond the scope of the present paper, simple inspection of the time series in vortex events shows that point-to-point variations of $5 \mathrm{~m} / \mathrm{s}$ are not unusual, and thus the rate of data acquisition alone limits the fidelity of the wind history to $\sim 3 \mathrm{~m} / \mathrm{s}$ or so (i.e. an arbitrary time shift of the reading by half a second would lead to a change in the value by this amount.) For the typical windspeeds of $5-20 \mathrm{~m} / \mathrm{s}$ in a vortex event, this seems broadly consistent with the stated accuracy of $15 \%$ (Banfield et al. 2019).

Lorenz (2016) described a model by which the diameter, intensity and miss distance of a vortex might be estimated (albeit not completely unambiguously) from pressure and wind time series. The intense fluctuations in InSight reported wind speeds, and the frequent data gaps, mean such an estimation is difficult in most of the encounters. Although it may succeed, perhaps in combination with seismometer data, in perhaps the best $10-20 \%$ of cases, this has not been attempted in the present work.

Dust devils have an optical signature, as their name implies, and can be detected by the solar shadow cast by the dust (e.g. Lorenz and Jackson, 2015). InSight's solar panels can in principle record such shadows, and these records have indicated dust variations and clouds on Mars (Lorenz et al., 2020). However, solar array data are only recorded when the lander is 'awake' to perform radio transmissions or other high power operations, hence long gaps exist when no data are available. Furthermore, for most of the mission, solar array currents have been recorded at a low cadence of $\sim 30$ s intervals or longer, so the solar array information in the present catalog has very limited value. However, the sampling cadence was recently (Sol 426) increased to $4 \mathrm{~s}$ hence these data will likely be of greater interest in any future catalog releases.

Both positive and negative air temperature perturbations have been noted in terrestrial vehicle encounters (e.g. Sinclair, 1973), depending on whether a wide vortex with a resolved ('cold') downdraft is penetrated by the encounter (equivalently, the dust devil passes directly over a fixed station, as at InSight) or not. Detecting such air temperature perturbations is even more difficult on Mars, where sensors take appreciable time to respond to the thin atmosphere. The maximum and minimum air temperatures in the record are reported in the table to permit an assessment of variation overall, but temperature signatures are much less consistent than pressure or wind.

\section{Population characteristics}

The catalog allows exploration of statistical properties of, and correlations between, the various parameters. The large number of events permits relations to be exposed against the obfuscating random variation of vortex characteristics and encounter geometries.

The full catalog of 853 events is available electronically (see appendix); the summary entries of a small subset (the 'top ten' in terms of pressure drop Sols 1-390) are presented in Table 1. Some basic details of some other events of note, such as those discussed in the literature, are provided in Table 2. The following sections discuss some overall features of the population.

\subsection{Pressure and wind speed statistics}

The cumulative plot of peak pressure excursions (dips) is shown in Fig. 4. As noted previously (Banfield et al., 2020; Spiga et al., 2020), the InSight vortex population is very abundant, with several times more detections per day than previous landing sites for which pressure data were available (i.e. excluding the Gusev crater site at which the Spirit rover imaged large numbers of very large dust devils), and with the largest events detected on Mars to date (event 119 on InSight Sol 65). At our 0.8 Pa threshold, 2-3 events per Sol are observed. 

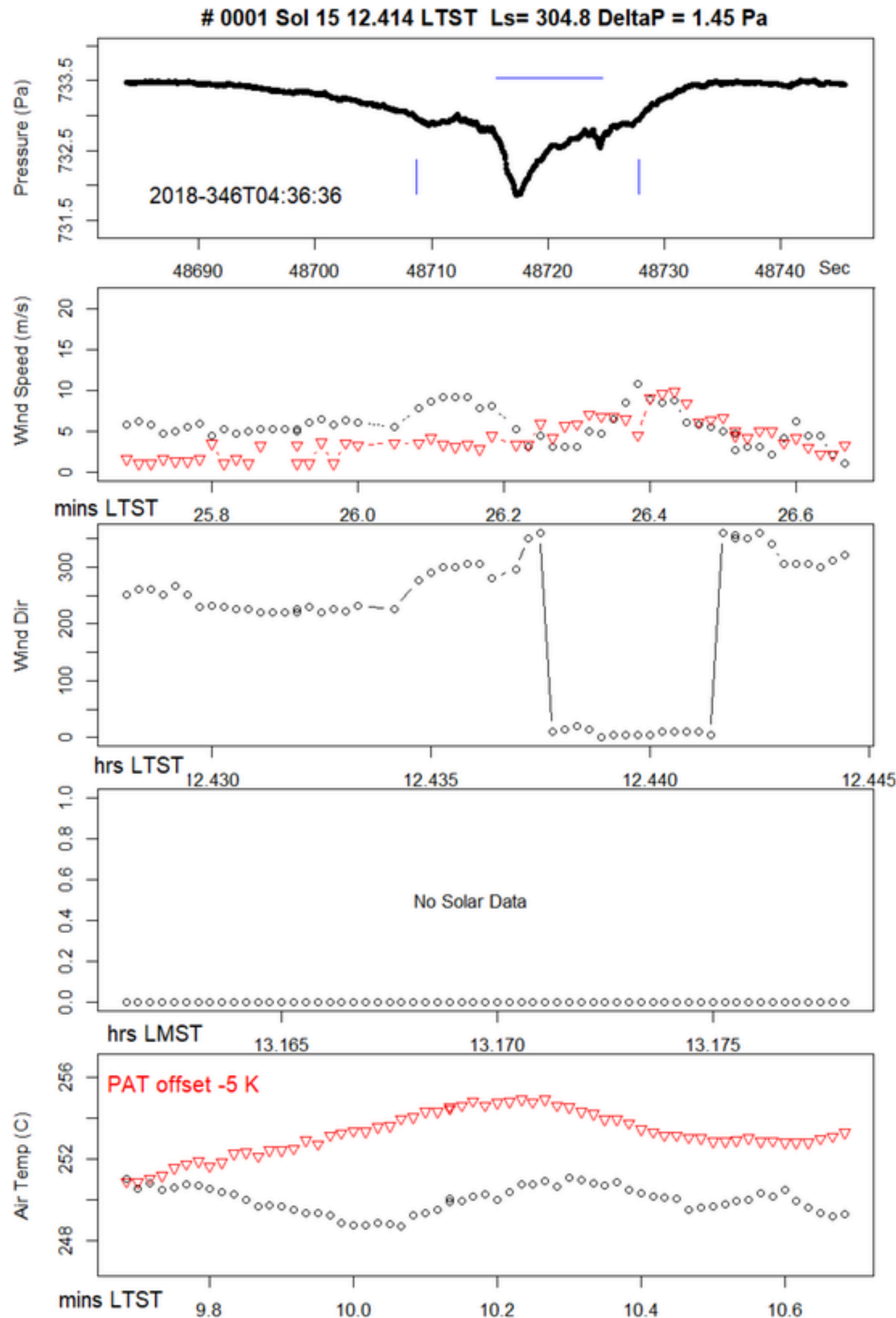

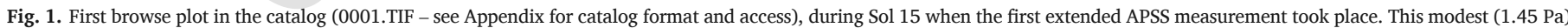

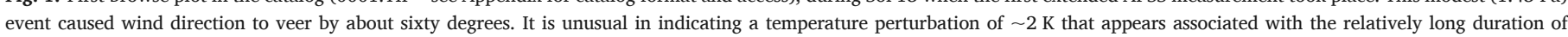

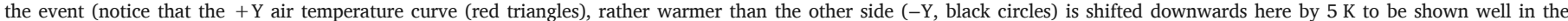

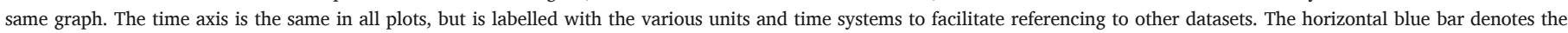

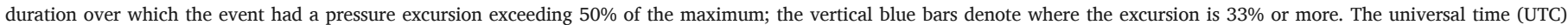




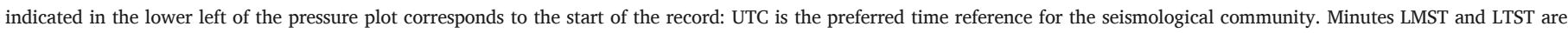

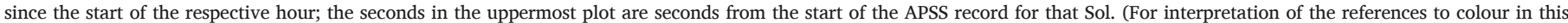
figure legend, the reader is referred to the web version of this article.)

The population has a skewed distribution that can be approximated by one or more power law segments. As discussed previously (Lorenz, 2013), lengthy but inconclusive debates may be had with finite, noisy datasets on the relative merits of power law, lognormal, exponential or other fits. In the present instance, an exponential (see e.g. Kurgansky, 2006) has a convexity similar to the population overall, but introduces too much curvature (as was the case for Mars Exploration Rover dust devil diameters, Lorenz, 2009, which were better fit by a power law). An alternate description may be of two power law segments, raising the interesting question of whether the break point between them (the 'knee' in the curve) at $3 \mathrm{~Pa}$ has any physical significance or interpretation. Spiga et al., 2020 show their catalog as differential rather than cumulative counts, and a change of slope can be discerned (albeit noisily) at this point. They fit the population with a single differential power law of -3.5 (thus a cumulative one of -2.5 , in effect a weighted average of the -2 and -4 slopes we identify here).

The peak observed pressure drop and its relation to the core pressure drop of the vortex, depends on the miss distance. Only in the case of a completely diametric encounter, where the center of the vortex passes right over the sensor (whose inlet is on the lander deck, Banfield et al., 2019) does the measurement recover the core pressure drop. Similarly, the peak wind speed measured is a vector sum of the ambient wind (assumed uniform) with the vortex wind field (zero at the core, and a maximum at the 'wall', and decaying with distance beyond the wall). As discussed in Lorenz (2016) and elsewhere, a wind time series that shows a single broad peak likely indicates an encounter that did not penetrate the wall (and thus did not sample the maximum wind), whereas a diametric encounter with a strong vortex will show a double peak as first the leading wall passes over the sensor, then the calm core, then the following wall. Although some modest uncertainties exist depending on the geometry, a good approximation of the maximum circumferential wind is the difference between the peak wind measured and the ambient wind (i.e. that before and after the encounter). We may note, however, that in many InSight encounters, the peak wind experienced by the lander may not be captured by the TWINS wind sensor record due to the highly dynamic nature of the flow defeating the pipeline calibration.

In principle, the peak wind and core pressure drop are related by the cyclostrophic balance: the radial pressure gradient $\mathrm{dP} / \mathrm{dr}$ at distance $\mathrm{r}$ provides the centripetal acceleration to cause the quasi-circular motion of the air parcel. Thus, $\mathrm{dP} / \mathrm{dr}=\rho \mathrm{V}^{2} / \mathrm{r}$ with $\mathrm{V}$ the local circumferential velocity and $\rho$ the atmospheric density. There are various analytic models of pressure and velocity profiles, but in all, the wall (the distance of maximum velocity) is where the pressure has fallen to one half of the core drop. Thus, depending on the function chosen, this integrates to $\Delta \mathrm{P}_{\text {core }}={\mathrm{K} \rho \mathrm{V}_{\text {max }}}^{2}$, with $\mathrm{K}$ in the range 1-2. Fig. 5 shows that the data are not inconsistent with this relationship (using $\mathrm{K} \sim 1$ and $\rho \sim 0.02 \mathrm{~kg}$ / $\mathrm{m}^{3}$, a typical value), albeit with large scatter since the observations may not capture the core pressure drop (due to non-diametric geometry), or may not capture the peak velocity (due to sensor drop-out or not penetrating the wall), or both (due to non-wall-penetrating geometry and/ or drop-out). Thus measured pressure drops serve as a useful proxy for peak speed and thus 'intensity' overall.

\subsection{Durations, area fraction and timing}

As noted in Jackson et al. (2018) the detection of transients by filtering (e.g. via the Short Term Average - Long Term Average difference or ratio (STA/LTA)) by definition pre-selects the duration of detected events. The filter used to detect events here and in Spiga et al., 2020 detects local drops in the 'detrended' pressure time series, i.e. that from which a 1000-s moving average has been subtracted. Thus this filter is in effect simply a high pass filter, which is effective here because the noise amplitude is lower than the events of interest. This approach would rejects events that are longer than several hundred seconds. However, in fact most events Figure 6are only about $10 \mathrm{~s}$ long. Fortunately the pressure sensor at least is generally sampled at a high rate (data recorded at up to 20 samples per second, although lower rates of 2 samples per second were used early in the mission) to provide good resolution even on these short events.

Of interest is the integrated duration of the detected events, about $6000 \mathrm{~s}$. If we consider the active period of convection to be roughly one quarter of the diurnal period long as on Earth (e.g. 11-17 h local solar time), then the observation period spans about 370 quarter sols or $\sim 8$ million seconds (although the Martian solar day or Sol is 88,775 s long, or $2.7 \%$ longer than Earth's, the rather approximate calculation here is not significantly affected), then the lander was 'inside' a vortex for $\sim 0.07 \%(7 \times 10-4)$ of the active period. This time fraction F (an 'exposure duty cycle') defines the effective instantaneous area fraction of vigorous dust devils (i.e. $>0.8 \mathrm{~Pa}$, or $>\sim 5 \mathrm{~m} / \mathrm{s}$ ) during the convective part of the day. The quantity is the integral across the vortex population $\mathrm{N}(\mathrm{D})$ per $\mathrm{km}^{2}$ per day of (diameter * longevity [t] * advection speed [U] $)^{1}$ times the duration of each event, i.e. (diameter / advection speed).

$\mathrm{F}=\Sigma \mathrm{N}(\mathrm{D}) *(\mathrm{DtU}) *(\mathrm{D} / \mathrm{U})=\Sigma \mathrm{N}(\mathrm{D}) \mathrm{D}^{2} \mathrm{t}$

A previous calculation, based on observed dust devil diameters obtained by imaging at the Gusev site by the Spirit rover, indicated an area fraction of $1.6 \times 10^{-4}$ (Lorenz and Jackson, 2016). However, this was calculated for the entire period (i.e. based on an optical diameter population and calculated over the full day). Thus it should be adjusted upwards by a factor of 4 to reflect the density during the convective part of the day, yielding a reassuring, if not remarkable, agreement $\left(\sim 6.4 \times 10^{-4}\right)$ with the InSight area fraction above.

The first term in eq.1, (DtU), is the area swept by the detectable pressure footprint of the vortex, and is defined in part by the pressure threshold used. If the pressure threshold is equivalent to the surface stress needed to lift surface material, then this $\Sigma \mathrm{N}(\mathrm{D}) \mathrm{DtU}$ quantity would become the track area generation rate in terms of $\mathrm{km}^{2}$ per $\mathrm{km}^{2}$ per day. Reiss and Lorenz (2016) measured this quantity in the InSight region to be $6 \times 10^{-5}$ to $3 \times 10^{-4} \mathrm{~km}^{2}$ per $\mathrm{km}^{2}$ per Sol, see also Perrin et al., 2020. If we multiply by the typical event duration $(\sim \mathrm{D} / \mathrm{U} \sim 7 \mathrm{~s}$ or $10^{-4}$ Sol, $4 \times 10^{-4}$ of the active period) then we find the area fraction $\mathrm{F}$ for track forming vortices to be only $10^{-7}$, or about four orders of magnitude smaller than the area fraction $\mathrm{F}$ we have calculated for vortices with $0.8 \mathrm{~Pa}$ pressure signatures. It is tempting to interpret Fig. 4 to infer, therefore, that the pressure drop which yields $10^{-4}$ of the encounter rate of 0.8 Pa vortices ( 20 per 10 Sols), i.e. 0.002 encounters per 10 sols, is the vortex pressure drop associated with track formation, implying about 20-50 Pa. The in-situ vortex data are consistent, then, with a dust-clearing/track-forming event occurring at a given spot only once per 5000 Sols or so at InSight. This long interval is in disappointing contrast with the 100-700 Sol interval and a 6-40 Pa threshold inferred by Lorenz and Reiss, 2015 for solar array cleaning events on the Spirit rover.

Quasi-periodicities have been noted in atmospheric vortex signatures on Earth and Mars, presumed to be due to the tendency of vor-

\footnotetext{
1 Note that an empirical relationship for longevity exists as a function of diameter, namely $t=40 \mathrm{D}^{0.66}$, where $t$ and $\mathrm{D}$ are in seconds and meters respectively (Lorenz, 2013).
} 

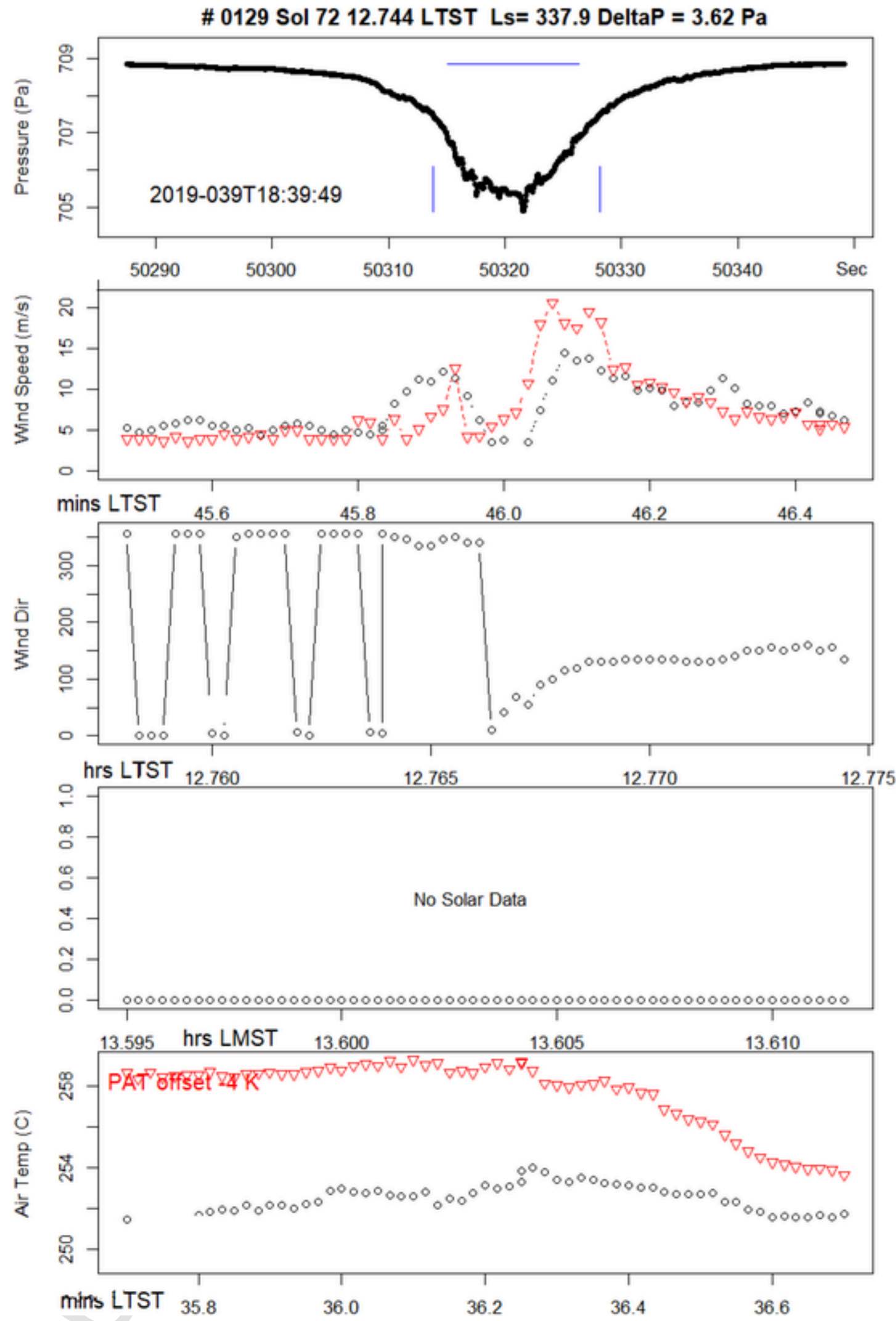

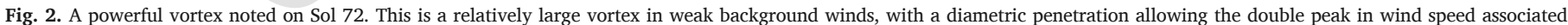
with the 'wall' to be resolved. The wind direction in the record is dominated by the vortex winds, with a before/after difference of about 150 degrees.

tices to form in the corners of the upwelling sheets of air in the quasi-regular cellular convection pattern in the planetary boundary layer. This can even manifest (Fenton and Lorenz, 2017) in four dust devils 

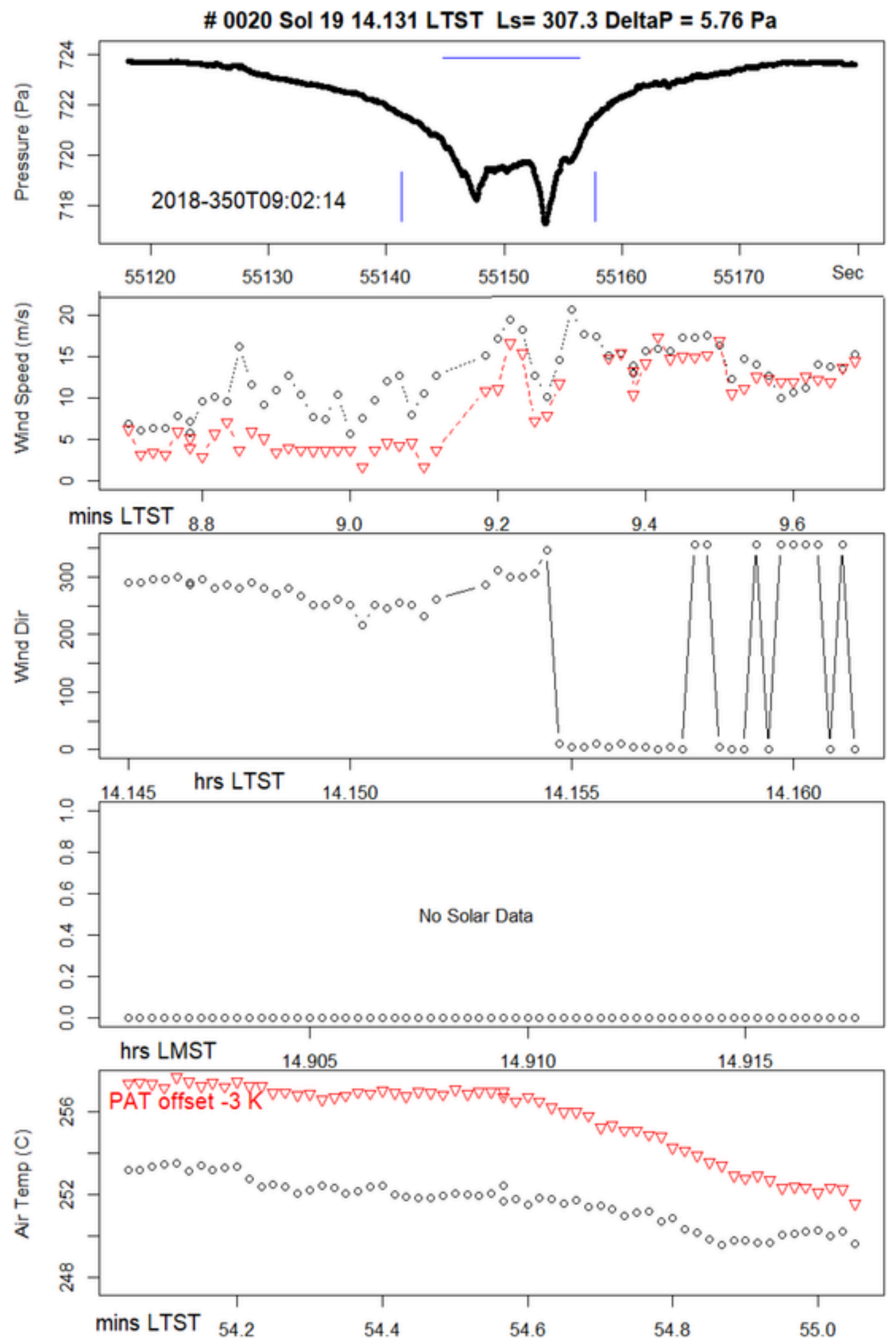

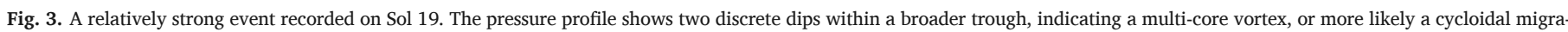
tion of a single core making two close encounters. 
Table 1

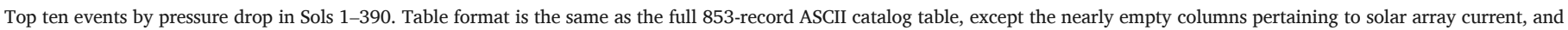
the LMST and UTC time columns have been omitted. The variables listed in each column are defined in the Appendix: a value of -9.9 indicates data are not available.

\begin{tabular}{|c|c|c|c|c|c|c|c|c|c|c|c|c|c|c|c|}
\hline Num & Sol & LTST & $\begin{array}{l}\Delta \mathrm{P} \\
(\mathrm{Pa})\end{array}$ & Nwind & $\begin{array}{l}\text { Wmax } \\
(\mathrm{m} / \mathrm{s})\end{array}$ & $\begin{array}{l}\text { Wbef } \\
(\mathrm{m} / \mathrm{s})\end{array}$ & $\begin{array}{l}\text { Waftr } \\
(\mathrm{m} / \mathrm{s})\end{array}$ & $\begin{array}{l}\text { Wdir } \\
\text { (deg) }\end{array}$ & $\begin{array}{l}\text { Atck } \\
\text { (s) }\end{array}$ & $\begin{array}{l}\text { Dcay } \\
\text { (s) }\end{array}$ & $\begin{array}{l}\text { Wdth } \\
\text { (s) }\end{array}$ & $\begin{array}{l}\text { MinT1 } \\
\text { (K) }\end{array}$ & $\begin{array}{l}\text { MaxT1 } \\
\text { (K) }\end{array}$ & $\begin{array}{l}\text { Press } \\
(\mathrm{Pa})\end{array}$ & $\begin{array}{l}\text { Ls } \\
\text { (deg) }\end{array}$ \\
\hline 00119 & 65 & 13.53 & 9.18 & 53 & 20.1 & 6.9 & 4.5 & 20 & 2.6 & 1.3 & 2.9 & 249.9 & 252 & 702.1 & 334 \\
\hline 00666 & 323 & 11.57 & 7.45 & 57 & 17.9 & 12 & 10.4 & 135 & 6 & 2.1 & 5.7 & 239 & 242.2 & 693.7 & 97.3 \\
\hline 00456 & 231 & 12.6 & 6.76 & 61 & 16.4 & 9.5 & 8 & 115 & 1.4 & 1.5 & 1.9 & 241 & 242.9 & 753.9 & 55.9 \\
\hline 00517 & 254 & 15.15 & 6.43 & 60 & 21.5 & 11 & 8.9 & 85 & 3.8 & 1.6 & 3.6 & 248.5 & 250.5 & 743.7 & 66.3 \\
\hline 00463 & 233 & 11.51 & 6.3 & 62 & 13.9 & 10.9 & 11.3 & 143 & 0.6 & 0.4 & 0.6 & 236.5 & 238 & 754.6 & 56.8 \\
\hline 00020 & 19 & 14.13 & 5.76 & 59 & 20.7 & 13.7 & 7.3 & 355 & 12.2 & 4.1 & 11.6 & 249.6 & 253.5 & 722.9 & 307.3 \\
\hline 00076 & 39 & 12.73 & 5.67 & 5 & 24.8 & -9.9 & -9.9 & 310 & 13.5 & 4 & 12 & 261 & 265.2 & 718.8 & 319.2 \\
\hline 00839 & 385 & 12.56 & 5.35 & 59 & 20 & 15.8 & 10.9 & 140 & 1.1 & 0.8 & 1.2 & 249.2 & 251.1 & 638.4 & 126.6 \\
\hline 00274 & 170 & 14.16 & 5.18 & 6 & 14 & -9.9 & -9.9 & 95 & 3.1 & 2.2 & 3.2 & 249.9 & 251.2 & 737 & 27.8 \\
\hline 00709 & 337 & 11.38 & 4.92 & 58 & 19.5 & 13.1 & 7.9 & 110 & 6.9 & 6.2 & 9.2 & 233.7 & 236.2 & 680 & 103.8 \\
\hline
\end{tabular}

appearing equispaced in a line. Cellular convective patterns were putatively detected by the ultra-sensitive pressure sensor on board InSight (Spiga et al., 2020), and regularly-spaced pressure dips have been noted in terrestrial studies.

If the boundary layer were a constant depth (say $5 \mathrm{~km}$ ), and advected at a constant speed $(10 \mathrm{~m} / \mathrm{s})$, then we would expect the roughly equant (depth/diameter $=1$ ) cells to pass at intervals of $\sim 500 \mathrm{~s}$ or $9 \mathrm{~min}$. If every cell wall was populated with a vortex, then encounters would occur with this period: on the other hand, if vortices only populate a subset of the cell boundaries, encounters would occur at integer multiples of the period.

A histogram of the intervals between events in the catalog is shown in Fig. 7. Although there are hints of peaks at $\sim 25$ and $50 \mathrm{~min}$, the significance is low. It may be that the changing depth of the boundary layer, and varying wind speeds, 'smear out' the peaks and that examination of restricted sets of data, and/or examination of the much more extensive catalog of pressure drops down to $0.3 \mathrm{~Pa}$, may show possible regularities better. An investigation of such regularities with LES would be of interest, but this is beyond the scope of the present paper.

\subsection{Vortex dependence on wind}

There are several aspects by which wind may influence vortex detections. First, it is well-known from optical field observations that there is a 'sweet spot' of wind speed for dust devil formation - some ambient wind is required to provide adequate circulation (or vorticity) for dust devil rotation. On the other hand, strong winds cause shear which tends to destroy the vortex structure: see discussion in Rafkin et al. (2016). There is also, as discussed in Spiga et al. (2020) an observation bias for single-station measurements towards higher detection numbers for a given formation rate, because (unless longevity has a dependence on wind speed, which may or may not be the case), a vortex sweeps a longer track and thus has a higher detection footprint, in stronger winds.

It is seen in Fig. 8 that most vortices at InSight were detected when the wind before the event was $7-13 \mathrm{~m} / \mathrm{s}$. However, this may be simply a result of afternoon winds at InSight being predominantly in this range (Spiga et al., 2020) - a more systematic study of the statistics of wind during non-detection periods would be needed to draw strong conclusions. Similarly, a histogram of wind azimuths (Fig. 9) shows that, consistent with the direction of dust devil tracks observed in the environs of the InSight landing site (Reiss and Lorenz, 2016; Perrin et al., 2020) most vortices were detected when the wind was blowing from the southeast $(\sim 140$ degrees) which is typical for the wind direction in the peak convection period of the day (afternoon) for this season. The secondary peak in the histogram is largely due to the early part of the mission when winds were more typically from the north or northwest.

\subsection{Asymmetry of pressure profiles}

In the ideal model of a two-dimensional vortex being advected past a measurement station (e.g. Lorenz, 2016), the pressure field is axisymmetric and the cut through it by the measurement site is bilaterally symmetric about the instant of closest approach. However, it has been noted (e.g. Sinclair, 1973; Lorenz, 2011) that the observed pressure profile is often asymmetric, with a shallower 'attack' and a steeper 'decay'. In the catalog, this shape aspect is quantified by separate attack and decay timescales (see appendix) defined by the times between the pressure excursion peak, and passing a threshold of $20 \%$ of the peak. We determine a skewness measure as the ratio of these two times, and this is plotted against windspeed in Fig. 1 (note that the number of points shown is less than the full catalog, since adequate wind data was not present for all events in the catalog.

It is seen that indeed, positively skewed (slower attack than decay) events are more common, by a factor of about three. The interpretation - as discussed in Sinclair (1973) is that the vortex is of course actually three-dimensional, and is tilted forward by the ambient wind shear. Crudely, ground ahead of the vortex intersection with the ground is closer to the tilted cylinder than is ground behind, and so sees part of the low pressure field earlier, hence the longer attack time. The scatter in Fig. 10 suggests that there may be some dependence of the skew on wind speed, with the stronger skew values occurring for intermediate to strong winds, although there may also be a fall-off at the highest ambient winds. The question may bear further study, perhaps with different skewness metrics and/or with Large-Eddy Simulations.

\subsection{Temperature fluctuations}

Warm near-surface air is the principal energy source for dust devils, and strong near-surface temperature gradients are a useful field criterion for vortex activity (e.g. Rafkin et al., 2016). The signature of a vortex in time series of temperatures at a fixed height is known to be challenging to recognize, and the correlation of temperature fluctuation with vortex intensity in the catalog (Fig. 11) is not especially strong.

Ground temperatures are recorded on InSight with a radiometer instrument (Mueller et al. 2020), intended principally to understand the surface boundary conditions on subsurface temperatures and heat flow. However, the data are not recorded frequently enough for routine comparison with vortices and thus is not included in this catalog, although since vortices are a manifestation of the sensible heat flux term in the surface energy balance (and a predictive metric of dust devil activity is 
Table 2

Catalog Events already discussed in the literature.

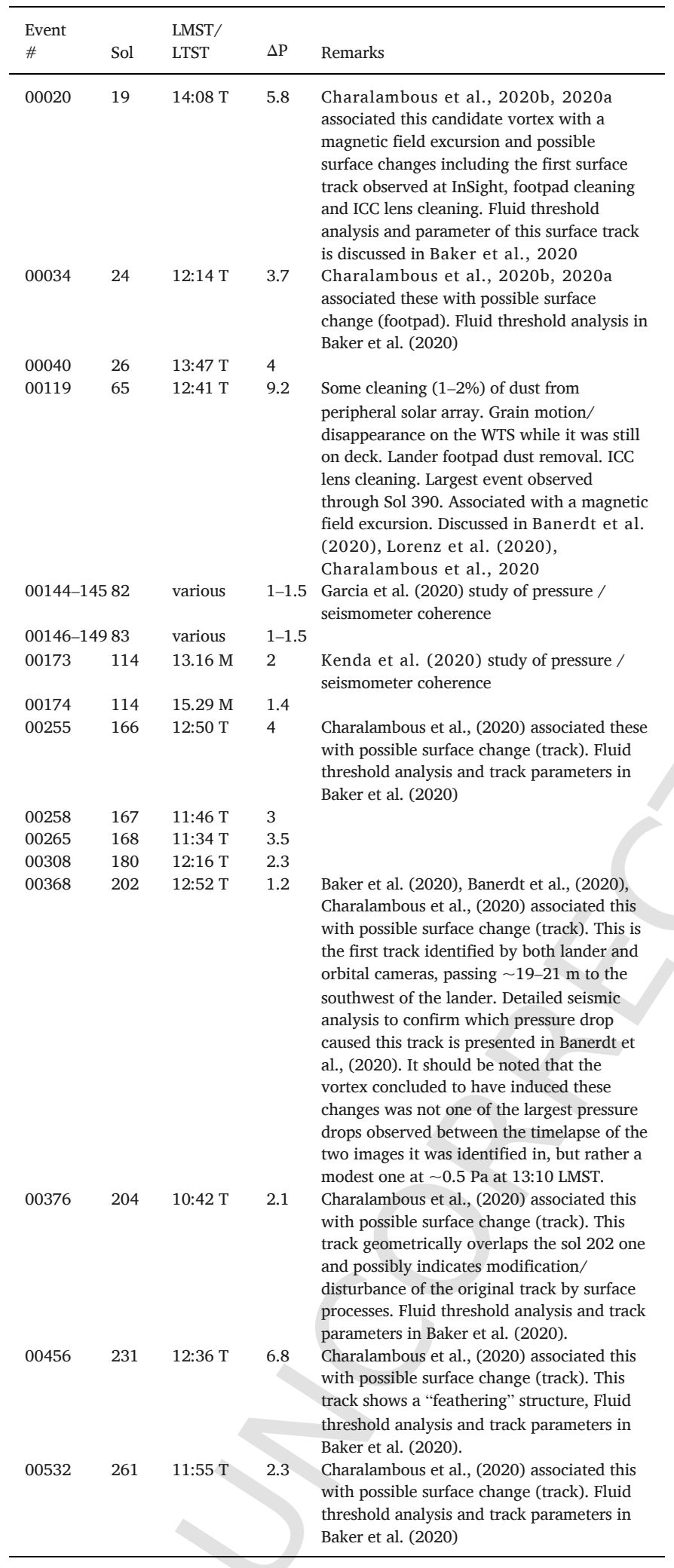

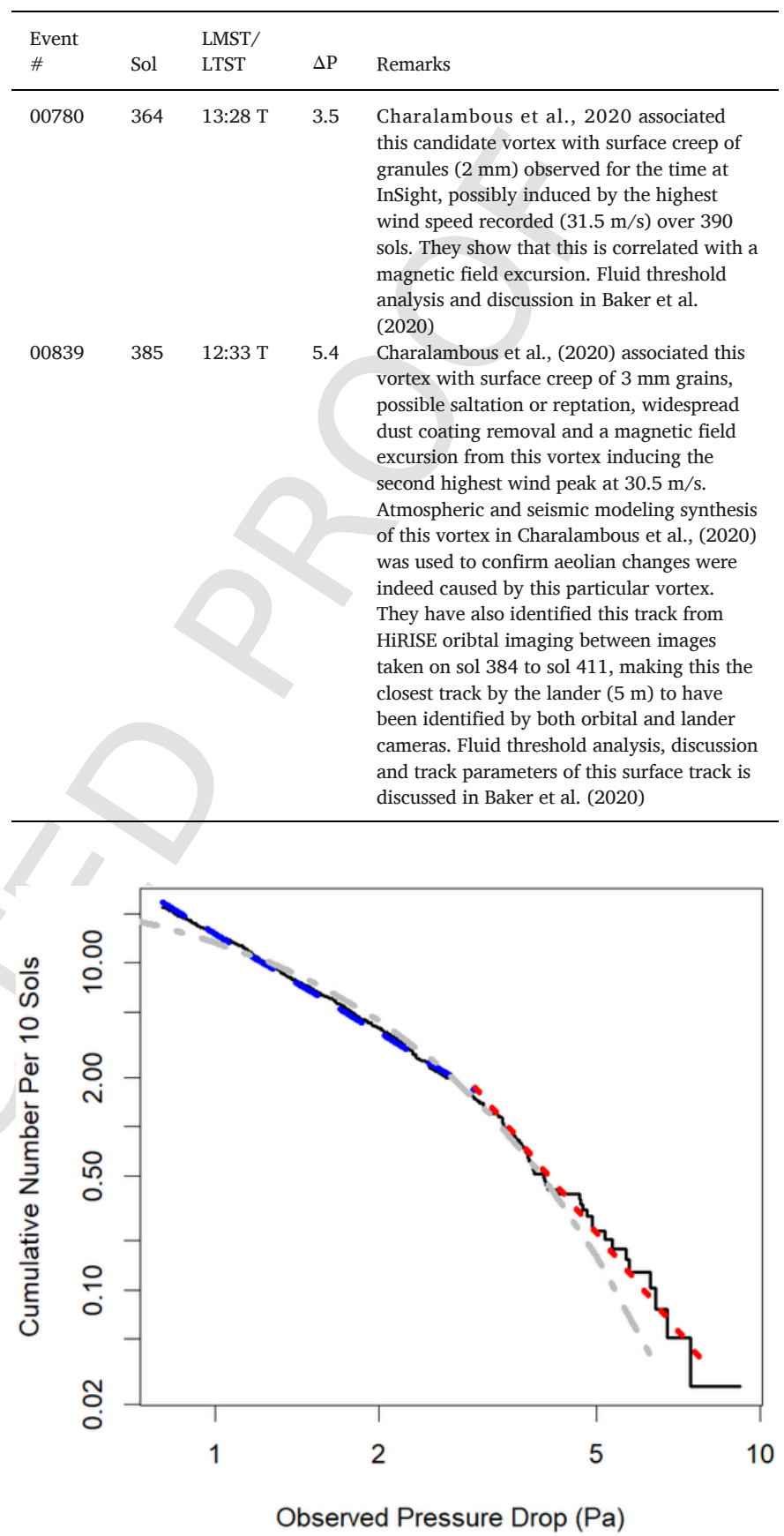

Fig. 4. The observed cumulative amplitude distribution of pressure drops. Below $3 \mathrm{~Pa}$, the data are very well described by a power law (blue dashed line) with a -2 slope: above $3 \mathrm{~Pa}$ where the raw counts (solid stepped line) become subject to statistical noise, the data are better fit with a - 4 slope (red dotted line). An exponential function (grey dash-dot) is shown for reference. (For interpretation of the references to colour in this figure legend, the reader is referred to the web version of this article.)

derived from this flux) a future examination of these data may be fruitful. Some seasonal trends on ground-air temperature differences and their influence on dust devil activity are noted by Spiga et al. (2020).

\subsection{Seismic signature}

Convective vortices (or more hopefully 'dust devils') were predicted before InSight launch to generate seismic signals. See field test on Earth by Lorenz et al. (2015) and modeling with Large Eddy Simulation and elastic loading theories by Kenda et al. (2017), Murdoch et al. (2017). This was verified by the first observations recorded by SEIS 


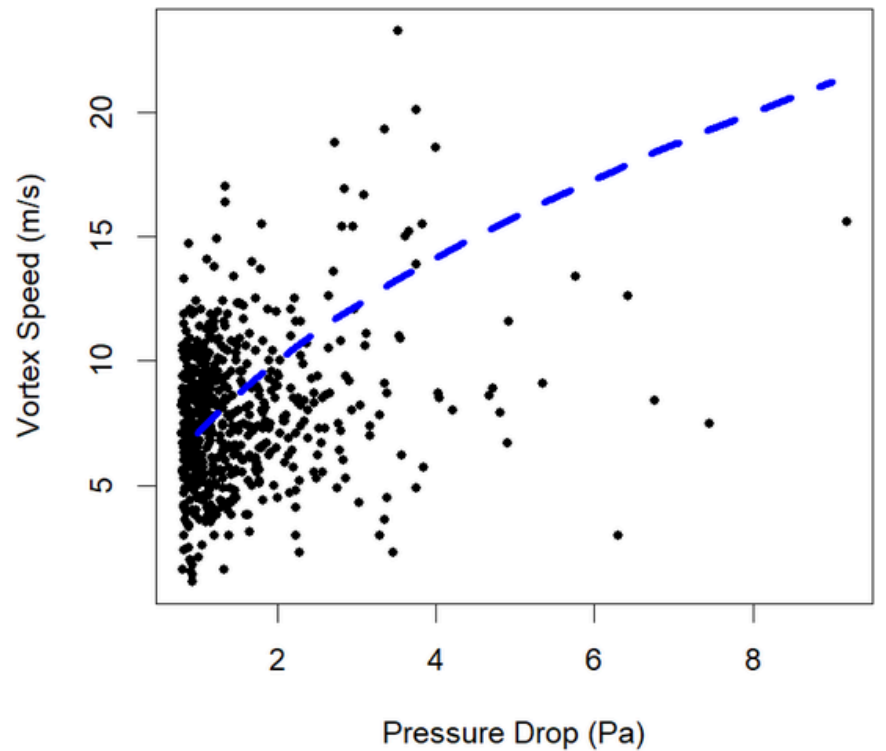

Fig. 5. Observed vortex speed and observed pressure drop. A reference curve with the expected $\mathrm{P} \sim \mathrm{K}_{\mathrm{N}} \mathrm{V}^{2}$ dependence (in this instance, $\mathrm{K} \rho=0.02 \mathrm{~kg} / \mathrm{m}^{3}$ ) is shown. Scatter results from the encounter geometry failing to sample the peak pressure drop, peak wind speed, or both.

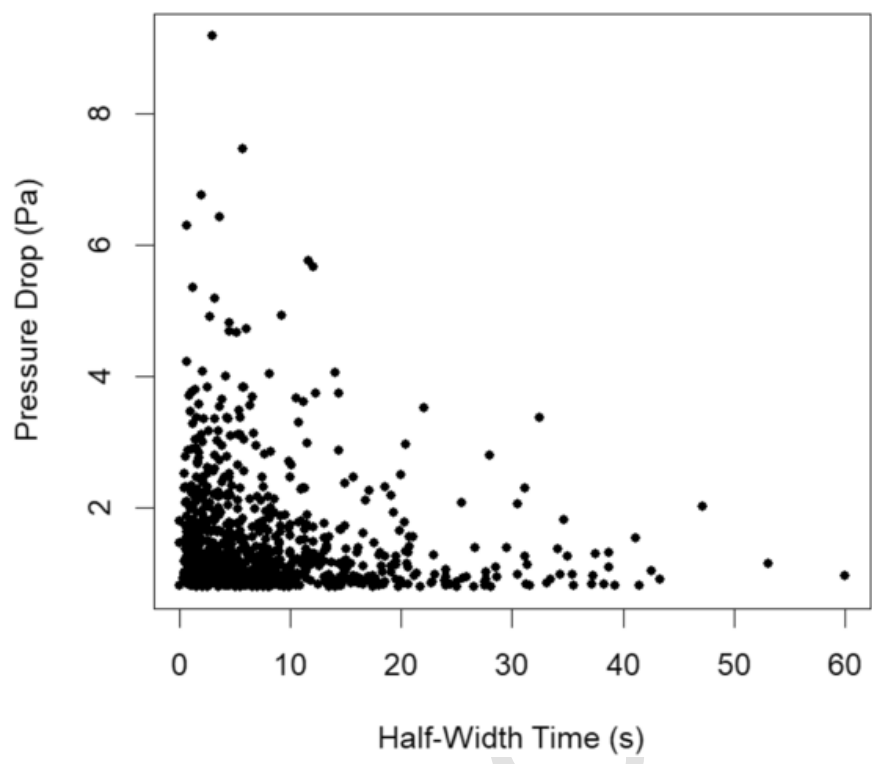

Fig. 6. Scatter plot of full width half-maximum duration versus recorded pressure drop. The majority of events, and all the largest ones ( $>5 \mathrm{~Pa}$ ) are less than $15 \mathrm{~s}$ long.

(Lognonné et al., 2019), as illustrated by Banerdt et al., 2020, Lognonné et al., 2020. A seismic signal is therefore a typical feature of many vortex encounters, although the waveforms of dust devils on SEIS SEIS Raw Data: InSight Mission, 2019might differ in shape (Fig. 12).

The seismic signal compliance defines the expected ground velocity response for a given pressure fluctuation. On the vertical axis it reaches typically $3-10 \times 10^{-7} \mathrm{~m} / \mathrm{s} / \mathrm{Pa}$ at $1 \mathrm{~Hz}$ depending on wind. For high wind, this compliance is increasing with frequency, as the pressure wave induced deformation is sensitive to the rigidity of the top most very low rigidity layers.

Although dust devils are mostly sources of long period, as already shown by several analysis (Kenda et al., 2020; Charalambous et al., 2020) they have significant energy remains at higher frequencies. Fig. 13 illustrates the energy at $2.4 \mathrm{~Hz}$, and suggests sensitivities of about

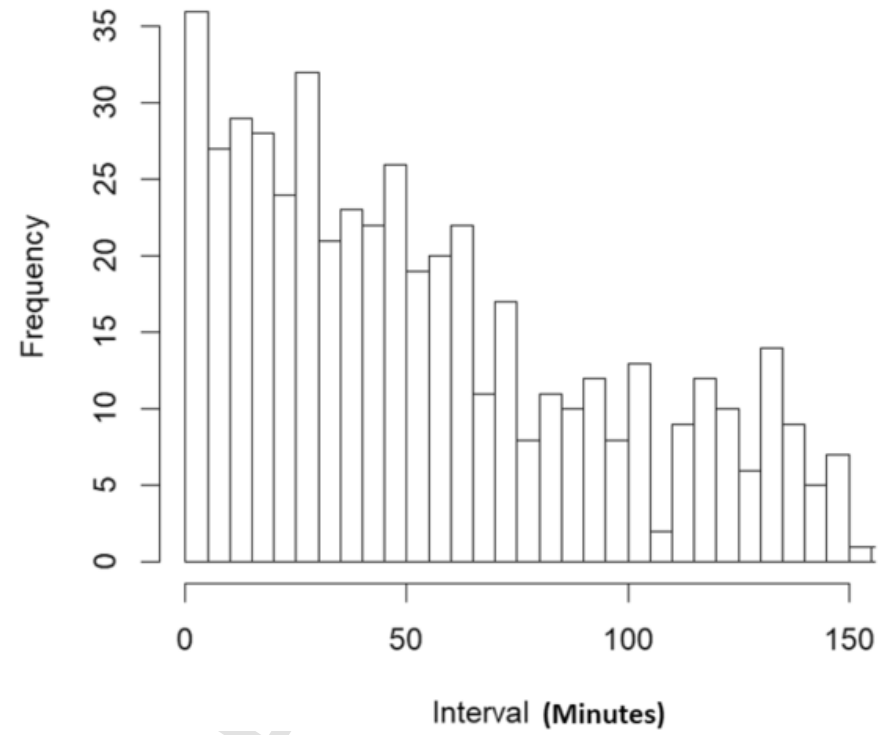

Fig. 7. Distribution of intervals (period from one vortex detection to the next).

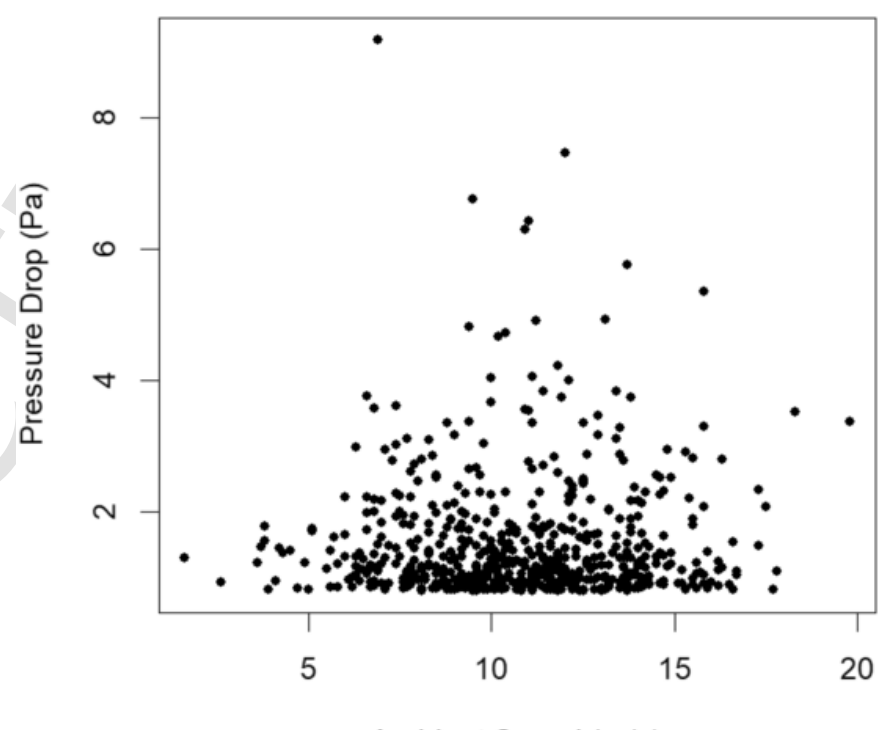

Ambient Speed (m/s)

Fig. 8. Most vortices overall, and most of the large ones, were detected when ambient winds were between $\sim 7$ and $13 \mathrm{~m} / \mathrm{s}$.

$2 \times 10^{-6} \mathrm{~m} / \mathrm{s} /$ Pa during dust devils. This is typically $2-7$ times larger than at $1 \mathrm{~Hz}$ and is compatible with a compliance increasing with both wind and frequency. Most likely, a significant part of the SEIS noise (Stutzmann et al., 2020), including to a few $\mathrm{Hz}$, is related to either dust devils resolved by the pressure sensors and possibly, at very noise level, to pressure drops or not resolved by the APSS sensor.

The maximum amplitudes of the SEIS unfiltered data (MSDS et al., 2019) as well as those at $2.4 \mathrm{~Hz}$ are reported in a separate supplementary data file to the catalog, when SEIS fast channels (10 sps or $20 \mathrm{sps}$ ) were available.

\section{Conclusions}

A catalog of vortex encounters has been presented, and has a range of applications both as an index to choose events for further study, and for statistical assessment of the population overall. The summary values in the catalog suggest asymmetric pressure profiles are somewhat common, with slower attack than decay slopes about twice as common, presumably due the asymmetry in the vortex induced by its advection. 


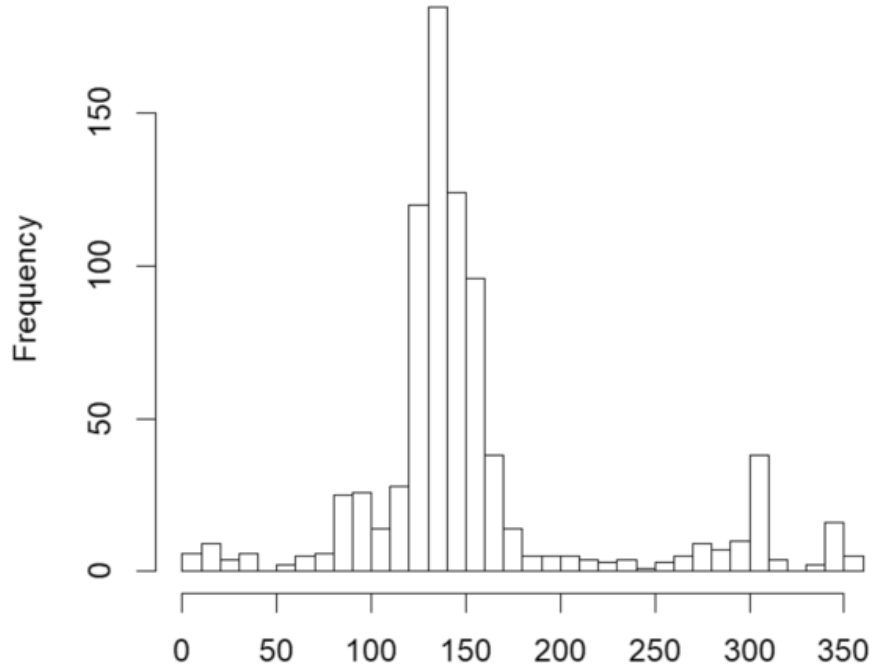

Wind Azimuth (Degrees)

Fig. 9. Wind azimuths prior to each event in the catalog. These likely reflect simply the preponderance of ambient wind directions during periods that favor vortex formation, not any preferential vortex formation rate as a function of wind direction.

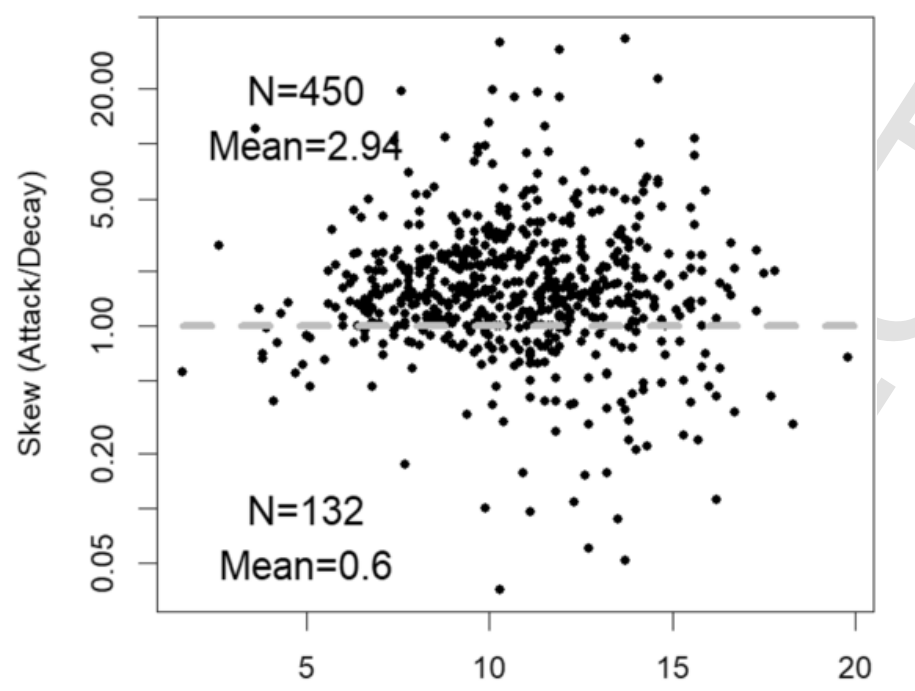

Wind Speed $(\mathrm{m} / \mathrm{s})$

Fig. 10. The skewness of the pressure profiles (attack timescale divided by decay). It is seen that most events have slower attack than decay. Note that the total number of points shown is less than the full catalog size, as only those entries with valid wind speeds are useful here.

As expected, vortices are a prominent feature of the seismometer background signals.

Uncited references

Banfield et al., 2019

SEIS Raw Data: InSight Mission, 2019

Declaration of Competing Interest

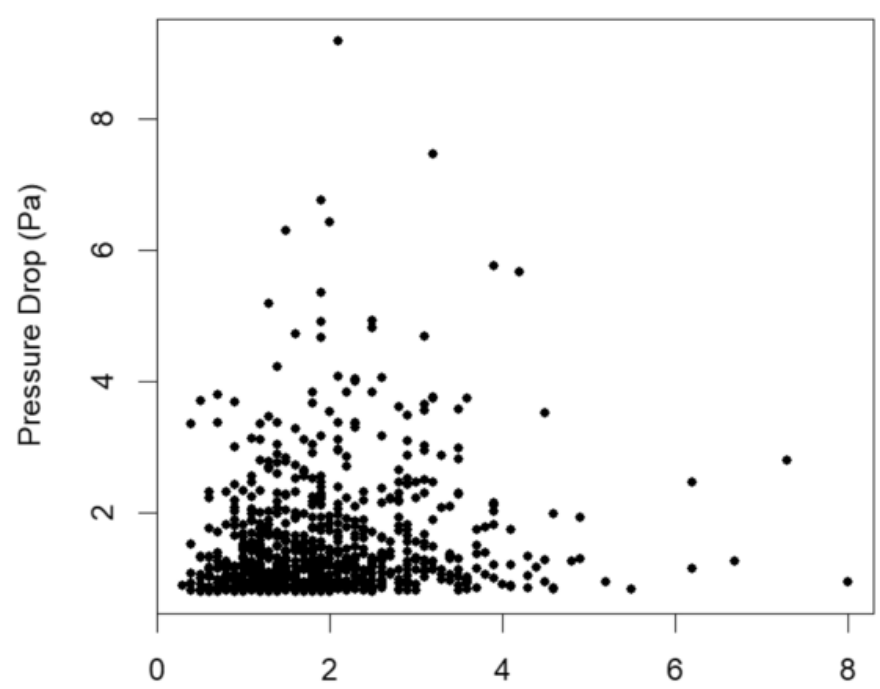

Temperature Variation (K)

Fig. 11. Relation of vortex intensity and the max-min air temperature range during the associated record.

\section{Acknowledgements}

This is InSight contribution number ICN 174. RL acknowledges the support of the NASA InSight Participating Scientist program via grant 80NSSC18K1626. French co-authors acknowledge support from Centre Nationale d'Études Spatiales (CNES) and Agence Nationale de la Recherche (ANR). CEN acknowledges the support of the NASA InSight Participating Scientist program via grant 80NSSC18K1630. We thank Daniel Viudez Moreiras and Sara Navarro for their work on and explanations of the TWINS wind data.

\section{Appendix A. Appendix}

The catalog has two elements: an ASCII table, and a zipped archive of TIFF images, comprising plots like Figs. 1-3 showing the details of each event. The two archive elements will be archived on the PDS: to permit community utilization pending the archival documentation and peer review phases, they are made available at the Applied Physics Laboratory Space data repository http://lib.jhuapl.edu/papers/the-dust-devilsof-elysium-vortex-catalog-and-mete/

After unzipping the archive it is easy to browse the collection of events. Detailed examination of individual events (identified in the table or via the images) can be performed by retrieval of the original APSS datafiles for the relevant Sol, archived at the PDS.

The catalog table comprises 21 columns, with entries as follows.

Number - this is the ordinal number in this catalog. There is a close, but not perfect, correspondence with Spiga's pressure drop list (Spiga et al., 2020), in that where incomplete wind data or other issues led to processing errors in catalog generation, those events were rejected. Only a handful of such eliminations were necessary - in this respect we estimate the catalog is $>98 \%$ complete with respect to possible pressure-only detections. Taking instrument anomalies and operating schedule (e.g. no operations in the first few days after landing) into account, the overall completeness is certainly $>95 \%$. The catalog numbers are ordinal in time (i.e. a lower number always preceded a higher number).

Sol - The InSight Sol number on which the event was observed.

LTST - Local True Solar Time, computed by using the position of the Sun and the landing site and recorded in the APSS files. Note that

None. 

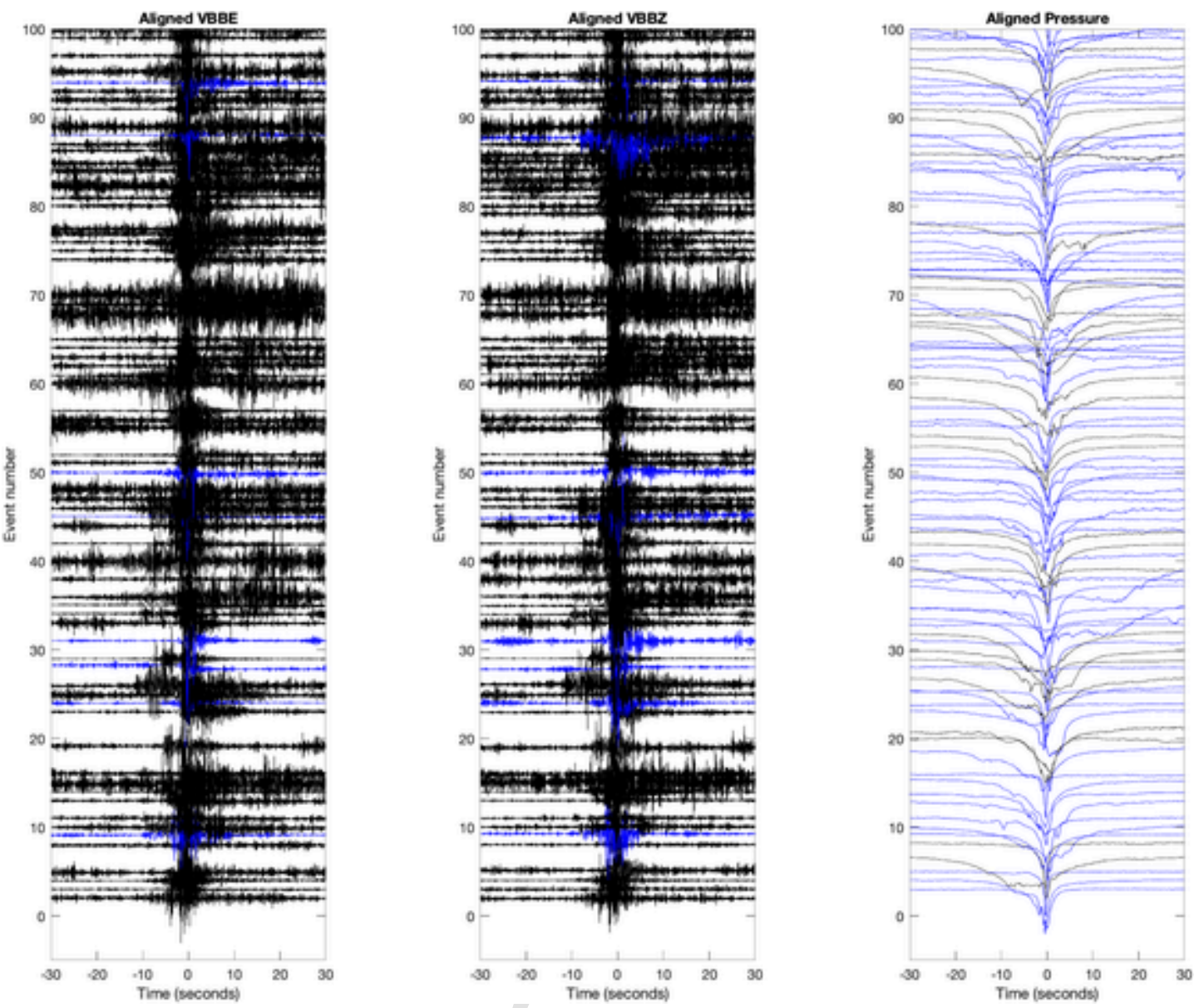

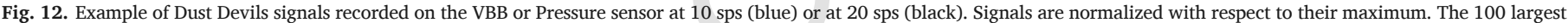

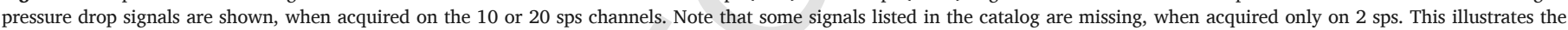

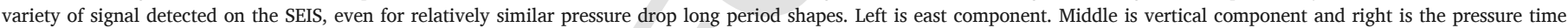
series. VBBE and VBBZ are in acceleration. (For interpretation of the references to colour in this figure legend, the reader is referred to the web version of this article.)

this time is the start of the record plotted in the browse image (i.e. approximately one minute before the peak pressure drop).

DP - The peak pressure drop in Pa.

Wmax - The maximum wind speed $(\mathrm{m} / \mathrm{s})$ recorded by either sensor during the record.

Wbef - The mean wind speed $(\mathrm{m} / \mathrm{s})$ in the $20 \mathrm{~s}$ at the start of the record (the higher of the Plus and Minus wind sensor means is recorded).

Waft - The mean wind speed (m/s)in the $20 \mathrm{~s}$ at the end of the record (the higher of the Plus and Minus wind sensor means is recorded).

Wazim - The mean wind direction (degrees from North) in the first 20 s of the record (i.e. before the vortex arrival).

Wvalid - Number of valid Plus wind measurements during the record.

Atck The interval in seconds between when the pressure drop exceeds $35 \%$ of its maximum value and when it reaches the maximum.

Dcay - The interval (s) etween the maximum pressure drop and when it relaxes back to less than $35 \%$ of maximum.

Wid - The duration of the pressure excursion in seconds that is more than $50 \%$ of the peak amplitude (i.e. the full width half maximum.)

Solar - The hard-tied solar array current in amps during the event (see Lorenz et al., 2020).
NSolar - The number of valid solar flux datapoints during the record.

Sdip - The percentage drop in solar flux associated with the dust devil. In the Sol 0-390 catalog this field is zero as there is insufficient data to estimate.

MinT1 - The minimum reading of the $-\mathrm{Y}$ wind sensor atmospheric temperatures during the record. The $+\mathrm{Y}$ wind sensor typically shows similar behavior during an encounter, but there is often an appreciable difference between the two, due to the wind-dependent perturbations of each by the warm lander deck and solar panels, so only one sensor $(-Y)$ is included in this catalog.

MaxT1 - The maximum reading of the $-\mathrm{Y}$ atmospheric temperatures during the event.

Press - The average absolute pressure value $(\mathrm{Pa})$ during the event. This allows the ambient density to be calculated.

Ls - The solar longitude of the event.

LMST The local mean solar time at the start of the record.

UTC The Universal Time at the start of the record.

Because the seismic amplitudes, from the two seismic instruments at often different sample rates, are only recovered for about a quarter of the pressure events in the catalog, the data shown in Fig. 13 are provided in a file separate from the main catalog. 


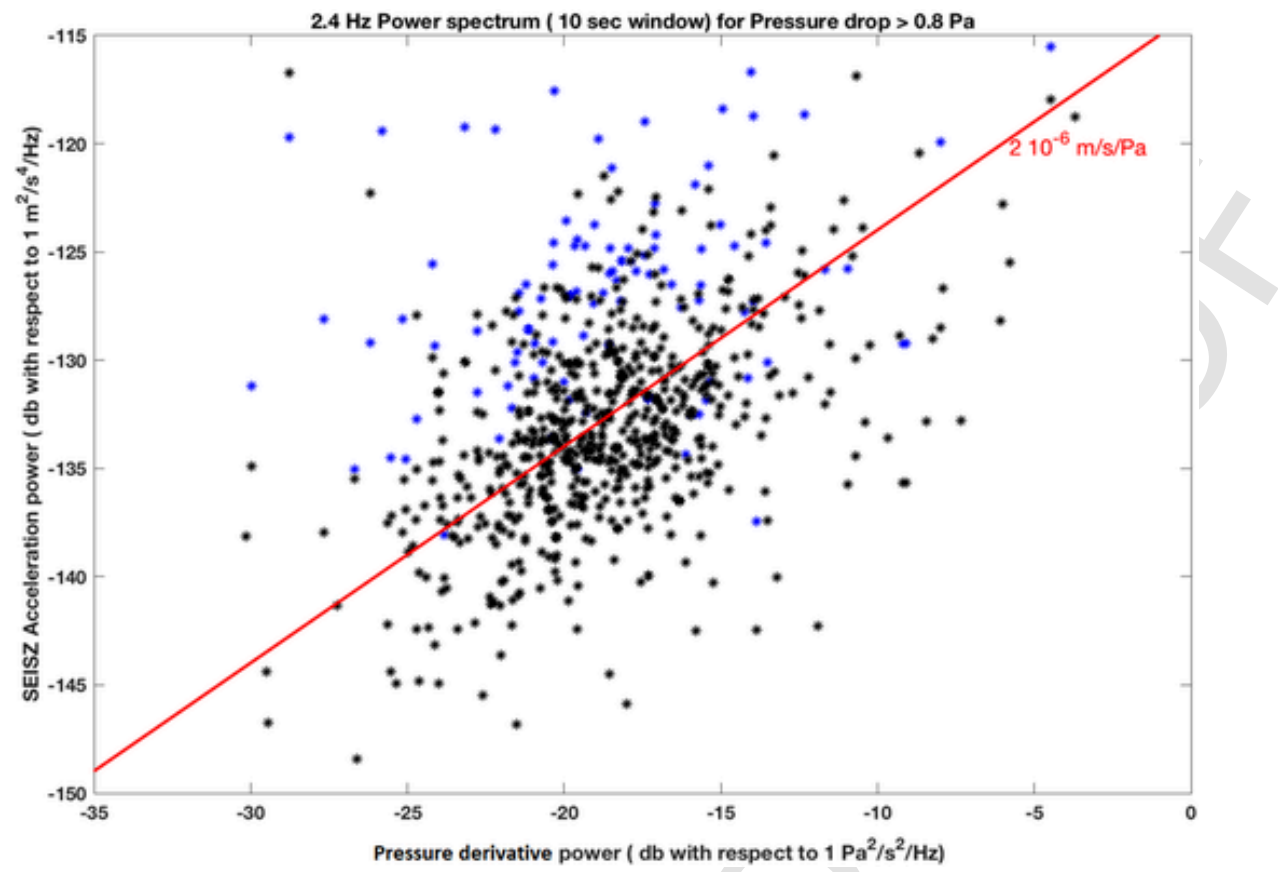

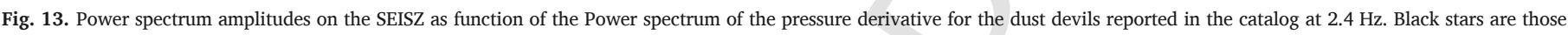

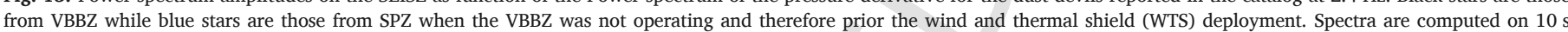

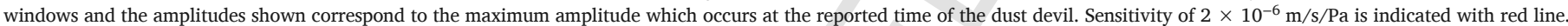

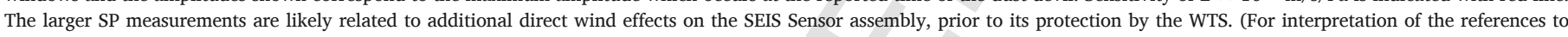
colour in this figure legend, the reader is referred to the web version of this article.)

\section{References}

Lognonné, P., Banerdt, W.B., Pike, W.T., Giardini, D., Christensen, U., Garcia, R.F., Kawamura, T., Kedar, S., Knapmeyer-Endrun, B., Margerin, L., Nimmo, F., Panning, M., Tauzin, B., Scholz, J.-R., Antonangeli, D., Barkaoui, S., Beucler, E., Bissig, F., Brinkman, N., Calvet, M., Ceylan, S., Charalambous, C., Davis, P., van Driel, M., Drilleau, M., Fayon, L., Joshi, R., Kenda, B., Khan, A., Knapmeyer, M., Lekic, V., McClean, J., Mimoun, D., Murdoch, N., Pan, L., Perrin, C., Pinot, B., Pou, L., Menina, S., Rodriguez, S., Schmelzbach, C., Schmerr, N., Sollberger, D., Spiga, A., Stähler, S., Stott, A., Stutzmann, E., Tharimena, S., Widmer-Schnidrig, R., Andersson, F., Ansan, V., Beghein, C., Böse, M., Bozdag, E., Clinton, J., Daubar, I., Delage, P., Fuji, N., Golombek, M., Grott, M., Horleston, A., Hurst, K., Irving, J., Jacob, A., Knollenberg, J., Krasner, S., Krause, C., Lorenz, R., Michaut, C., Myhill, B., Nissen-Meyer, T., ten Pierick, J., Plesa, A.-C., Quantin-Nataf, C., Robertsson, J., Rochas, L., Schimmel, M., Smrekar, S., Spohn, T., Teanby, N., Tromp, J., Vallade, J., Verdier, N., Vrettos, C., Weber, R., Banfield, D., Barrett, E., Bierwirth, M., Calcutt, S., Compaire, N., Johnson, C., Mance, D., Euchner, F., Kerjean, L., Mainsant, G., Mocquet, A., Manfredi, J. Antonio Rodriguez, Pont, G., Laudet, P., Nebut, T., de Raucourt, S., Robert, O., Russell, C.T., Sylvestre-Baron, A., Tillier, S., Warren, T., Wieczorek, M., Yana, C., Zweifel, P., 2020. Constraints on the shallow elastic and anelastic structure of Mars from InSight seismic data. Nat. Geosci. in press. doi:10.1038/s41561-020-0536-y.

Banfield, D., Rodriguez-Manfredi, J.A., Russell, C.T., Rowe, K.M., Leneman, D., Lai, H.R., Cruce, P.R., Means, J.D., Johnson, C.L., Mittelholz, A., Joy, S.P., 2019. Insight auxiliary payload sensor suite (apss). Space Sci. Rev. 215 (1), 4. doi:10.1007/ s11214-018-0570-x.

Banfield, D., Spiga, A., Newman, C., Forget, F., Lemmon, M., Lorenz, R., Murdoch, N., Viudez-Moreiras, D., Pla-Garcia, J., Garcia, R.F., Lognonné, P., Perrin, C., Martire, L., Teanby, N., Maki, J., Kenda, B., Mueller, N., Rodriguez, S., Kawamura, T., McClean, J., Stott, A., Charalambous, C., Millour, E., Johnson, C., Mittelholz, A., Clinton, J., Stähler, S., Giardini, D., Warren, T., Pike, T., Daubar, I., Golombek, M., Rolland, L., Widmer Schnidrig, R., Mimoun, D., Beucler, E., Lucas, A., Baker, M., Ansan, V., Karatekin, O., Lewis, S., Hurst, K., Mora Sotomayor, L., Navarro, S., Torres, J., Lepinette, A., Molina, A., Marin Jimenez, M., Gomez-Elvira, J., Peinado, V., Rodriguez-Manfredi, J.-A., Carcich, B., Sackett, S., Russell, C., Spohn, T., Smrekar, S., Banerdt, W.B., 2020. The atmosphere of Mars as observed by InSight. Nat. Geosci.. doi:10.1038/s41561-020-0534-0.

Cantor, B.A., Kanak, K.M., Edgett, K.S., 2006. Mars orbiter camera observations of Martian dust devils and their tracks (September 1997 to January 2006) and evaluation of theoretical vortex models. J. Geophys. Res. Planets 111 (E12). doi:10.1029/ 2006JE002700.

Charalambous, C., McClean, J., Baker, M.M., Pike, T., Golombek, M.P., Lemmon, M.T., et al., 2020a. Aeolian changes at the Insight landing site on mars: multi-instrument observations. Earth Space Sci. Open Arch. 17. doi:10.1002/essoar.10503003.1.

Charalambous, C., Stott, A.E., Pike, T., McClean, J., Warren, T., Spiga, A., Banfield, D., Garcia, R.F., Clinton, J., Stähler, S.C., López, S. Navarro, Lognonné, P., Kawamura, T., van Driel, M., Böse, M., Ceylan, S., Khan, A., Horleston, A.C., Orhand-Mainsant,
G., Sotomayor, L. Mora, Murdoch, N., Giardini, D., 2020b. A comodulation analysis of atmospheric energy injection into the ground motion at InSight, mars. Earth Space Sci. Open Arch. 48. doi:10.1002/essoar.10503206.1.

Ellehoj, M.D., Gunnlaugsson, H.P., Taylor, P.A., Kahanpää, H., Bean, K.M., Cantor, B.A., Gheynani, B.T., Drube, L., Fisher, D., Harri, A.M., Holstein-Rathlou, C., 2010. Convective vortices and dust devils at the Phoenix Mars mission landing site. J. Geophys. Res. Planets 115 (E4). doi:10.1029/2009JE003413.

Holstein-Rathlou, C., Gunnlaugsson, H.P., Merrison, J.P., Bean, K.M., Cantor, B.A., Davis, J.A., Davy, R., Drake, N.B., Ellehoj, M.D., Goetz, W., Hviid, S.F., 2010. Winds at the Phoenix landing site. J. Geophys. Res. Planets 115 (E5). doi:10.1029/2009JE003411.

Jackson, B., Lorenz, R., Davis, K., 2018. A framework for relating the structures and recovery statistics in pressure time-series surveys for dust devils. Icarus 299, 166-174.

Kahre, M.A., Murphy, J.R., Haberle, R.M., 2006. Modeling the Martian dust cycle and surface dust reservoirs with the NASA Ames general circulation model. J. Geophys. Res. Planets 111 (E6). doi:10.1029/2005JE002588.

Kenda, B., P.Lognonné, A.Spiga, T.Kawamura, S.Kedar, W. Bruce Banerdt, R.Lorenz, Modeling of ground deformation and shallow surface waves generated by Martian dust, devils and perspectives for near-surface structure inversion, Space Sci. Rev., 211, 501-524, Doi: https://doi.org/10.1007/s11214-017-0378-0, 2017.

Kenda, B., Drilleau, M., Garcia, R.F., Kawamura, T., Murdoch, N., Compaire, N., et al., 2020. Subsurface structure at the InSight landing site from compliance measurements by seismic and meteorological experiments. J. Geophys. Res. Planets 125, e2020JE006387. doi:10.1029/2020JE006387.

Kinch, K.M., et al., 2015. Dust deposition on the decks of the Mars exploration rovers: 10 years of dust dynamics on the panoramic camera calibration targets. Earth Space Sci. 2 (5), 144-172.

Kurgansky, M.V., 2006. Steady-state properties and statistical distribution of atmospheric dust devils. Geophys. Res. Lett. 33 (19), 2006GL026142.

Lognonné, P., Banerdt, W.B., Giardini, D., et al., 2019. SEIS: Insight's seismic experiment for internal structure of Mars. Space Sci. Rev. 215 (12). doi:10.1007/ s11214-018-0574-6.

Lorenz, R.D., 2009. Power law of dust devil diameters on earth and Mars. Icarus 203, 683-684.

Lorenz, R.D., 2011. On the statistical distribution of dust devil diameters. Icarus 215, 381-390.

Lorenz, R.D., 2013. The longevity and aspect ratio of dust devils: effects on detection efficiencies and comparison of landed and orbital imaging at Mars. Icarus 226, 964-970.

Lorenz, R.D., 2016. Heuristic estimation of dust devil vortex parameters and trajectories from single-station meteorological observations: application to InSight at Mars. Icarus 271, 326-337.

Lorenz, R.D., Jackson, B.K., 2015. Dust devils and dustless vortices on a desert playa observed with surface pressure and solar flux logging. GeoResJ 5, 1-11.

Lorenz, R.D., Jackson, B.K., 2016. Dust devil populations and statistics. Space Sci. Rev. 203, 277-297. doi:10.1007/s11214-016-0277-9.

Lorenz, R.D., Kedar, S., Murdoch, N., Lognonné, P., Kawamura, T., Mimoun, D., Banerdt, W.B., 2015. Seismometer detection of dust devil vortices by ground tilt. Bull. Seismol. Soc. Am. 105, 3015-3023. doi:10.1785/0120150133. 
Lorenz, R.D., Lemmon, M.T., Maki, J., Banfield, D., Spiga, A., Charalambous, C., Barrett, E., Herman, J.A., White, B.T., Pasco, S., Banerdt, W.B., 2020. Scientific observations with the InSight solar arrays: dust, clouds and eclipses on Mars. Earth Space Sci.. doi:10.1029/2019EA000992.

Lorenz, Ralph, Reiss, Dennis, 2015. Solar Panel Clearing Events, Dust Devil Tracks, and in-situ Vortex Detections on Mars. Icarus 248, 162-164.

Murdoch, N., Kenda, B., Kawamura, T., Spiga, A., Lognonné, P., Mimoun, D., Banerdt, W.B., 2017. Estimations of the seismic pressure noise on Mars determined from Large Eddy Simulations and demonstration of pressure decorrelation techniques for the InSight mission. Space Sci. Rev. 211, 457-483. doi:10.1007/s11214-017-0343-y.

Murphy, J.R., Nelli, S., 2002. Mars Pathfinder convective vortices: frequency of occurrence. Geophys. Res. Lett. 29 (23), 18-21.

Ordóñez-Etxeberria, I., Hueso, R., Sánchez-Lavega, A., 2020. Strong increase in dust devil activity at Gale crater on the third year of the MSL mission and suppression during the 2018 global dust storm. Icarus 113814.

Perrin, C., Rodriguez, S., Jacob, A., Lucas, A., Spiga, A., Kawamura, T., Murdoch, N., Pan, L., Lorenz, R., Daubar, I.J., Lognonne, P., Banfield, D., Banks, M.E., Garcia, R.F., Newman, C., Ohja, L., Widmer-Schnidrig, R., Banerdt, W.B., 2020. Monitoring of dust devil tracks around the InSight landing site, mars, and comparison with in-situ atmospheric data. Geophys. Res. Lett. 47 (10), e2020GL087234. doi:10.1029/ 2020GL087234.

Rafkin, S., Jemmett-Smith, B., Fenton, L., Ito, J., Lorenz, R., Tyler, D., 2016. Dust devil formation. Space Sci. Rev. 203, 187-207.

Reiss, D., Lorenz, R., 2016. Dust devil track survey at Elysium Planitia, Mars: implications for the InSight landing sites. Icarus 266, 315-330.

SEIS Raw Data: InSight Mission, 2019. InSight Mars SEIS Data Service, IPGP, JPL, CNES, ETHZ, ICL, MPS, ISAE-Supaero, LPG, MSFC. doi:10.18715/SEIS.INSIGHT.XB 2016.
Sinclair, P.C., 1973. The lower structure of dust devils. J. Atmos. Sci. 30 (8), 1599-1619. Baker, M, Newman, C, Charalambous, C, et al., 2020. Evidence for vortex-dominated aeolian activity at InSight's landing site. JGR-Planets. Submitted for publication.

Banerdt, W.B., Smrekar, S., Banfield, D., Giardini, D., Golombek, M., Johnson, C., Lognonné, P., Spiga, A., Spohn, T., Stähler, S., Antonangeli, D., Asmar, S., Beghein, C., Bowles, N., Bozdag, E., Chi, P., Christensen, U., Clinton, J., Collins, G., Daubar, I., Dehant, V., Fillingim, M., Folkner, B., Garcia, R., Garvin, J., Grant, J., Grott, M., Grygorczuk, J., Hudson, T., Irving, J., Khan, A., Kargl, G., Kawamura, T., Kedar, S., King, S., Knapmeyer-Endrun, B., Knapmeyer, M., Lemmon, M., Lorenz, R., Maki, J., Margerin, L., McLennan, S., Michaut, C., Mimoun, D., Mittelholz, A., Mocquet, A., Morgan, P., Mueller, N., Nagihara, S., Newman, C., Nimmo, F., Panning, M., Pike, T. Plesa, A.-C., Rodriguez-Manfredi, J.-A., Russell, C., Schmerr, N., Siegler, M., Stanley, S., Teanby, N., Tromp, J., van Driel, M., Warner, N., Weber, R., Wieczorek, M., 2020. Early results from the InSight mission: mission overview and global seismic activity. Nat. Geosci.. doi:10.1038/s41561-020-0544-y.

A. Spiga et al. https://arxiv.org/abs/2005.011342020

Stutzmann, E., Schimmel, M., Lognonné, P., Horleston, A.C., Ceylan, S., van Driel, M., Stähler, S.C., Banerdt, W.B., Calvet, M., Charalambous, C., Charalambous, C., Clinton, J., Drilleau, M., Fayon, L., Garcia, R.F., Giardini, D., Hurst, K., Jacob, A., Kawamura, T., Margerin, L., Murdoch, N., Pike, T., Scholz, J.-R., Spiga, A., 2020. Polarized ambient noise on mars. Earth Space Sci. Open Arch. 41. doi:10.1002/ essoar.10503376.1.

Vicente-Retortillo, Á., Martínez, G.M., Renno, N., Newman, C.E., Ordonez-Etxeberria, I., Lemmon, M.T., Richardson, M.I., Hueso, R., Sánchez-Lavega, A., 2018. Seasonal deposition and lifting of dust on mars as observed by the Curiosity rover. Sci. Rep. 8 (1), 1-8. doi:10.1038/s41598-018-35946-8. 